\title{
Intraocular pressure in a cohort of healthy eastern European schoolchildren: variations in method and corneal thickness
}

Patrycja Krzyżanowska-Berkowska', Magdalena Asejczyk-Widlicka² and Barbara Pierscionek ${ }^{3^{*}}$

\begin{abstract}
Background: Intraocular pressure (IOP) in the developing eye of a child is not always easy to measure and there is no technique that is known to be the most accurate for the young eye. Measurements are needed on many cohorts of children with different tonometers to determine how the values correlate between instruments, whether corneal parameters affect readings and whether correlations between age and IOP values can be discerned. The aim of this study was to undertake a comparative analysis of three different tonometers on a group of healthy children to see whether differences exist and whether these may be related to central corneal thickness and/or radius of curvature. In addition, the study adds to the relatively small body of literature on IOP in the growing eye which will collectively allow trends to be identified and ultimately norms to be established.
\end{abstract}

Methods: IOP was measured on 115 eyes in a group of Polish children, aged between $5-17$ years (mean \pm standard deviation [SD] $11.3 \pm 3.0$ years) using three different tonometers: non-contact (NCT), the ICare and Goldmann applanation (GAT). Readings obtained were compared between instruments and with central corneal thickness and radius of curvature.

Results: The ICare tonometer provided statistically higher IOP values $(16.9 \pm 3.4 \mathrm{mmHg})$ than the GAT (14.7 \pm $2.9 \mathrm{mmHg}$ ) regardless of corneal thickness and whether or not a correction factor was applied. A correlation was found between central corneal thickness (CCT) and IOP values obtained with all three tonometers but only the IOP values detected with the ICare tonometer showed a statistically significant correlation with radius of curvature $(p<0.004)$. No correlations with age or gender were found for IOP values measured with any of the instruments.

Conclusions: IOP measurements on children vary significantly between instruments and correlations are affected by the corneal thickness. Further studies on children are needed to determine which instrument is most appropriate and to derive a normative IOP scale for the growing eye.

Keywords: Cornea, Child, Paediatric Ophthalmology, Intraocular pressure, Tonometry

\section{Background}

An accurate, reliable and repeatable measure of intraocular pressure (IOP) is one of the fundamental requirements for glaucoma diagnosis and applanation tonometry remains the most commonly used and trusted technique in clinical practice. In children, however, standard methods such as applanation tonometry may be difficult to administer and consequently yield inaccurate or erroneous results [1-6].

\footnotetext{
* Correspondence: b.pierscionek@kingston.ac.uk

${ }^{3}$ Faculty of Science, Engineering and Computing, Kingston University, (Roehampton Vale Campus), Friars Avenue, London SW15 3DW, UK Full list of author information is available at the end of the article
}

Whilst a number of studies comparing IOP measurements with the ICare tonometer with applanation tonometry and other methods have been published, these have been conducted mainly in adults, a proportion of whom were patients diagnosed with glaucoma [7-13]. A relatively small proportion of the literature is devoted to measurements of IOP in children. Even fewer studies have described measurements, on young eyes, using the ICare tonometer.

The growing and developing eye in a child is different compared to the fully grown and therefore more stable eye of the adult. Consequently, the standard normal

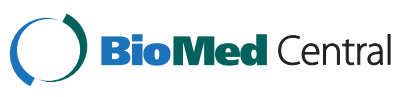


range of IOP in adults cannot be used for children. Measurements on large populations of children, without eye disease or conditions that may affect IOP, from a variety of countries and ethnic groups is required, to determine the standard range of IOP values applicable for every age of the growing eye.

The measurement of IOP in children yields difficulties that are not associated with tonometry on adult eyes. In addition to the issues that relate to attention, understanding of the procedure and co-operation with the examination, that can render contact methods difficult and measurements inaccurate, there are the physiological differences in ocular biometry and its proportions between the adult system and growing eyes of children.

The aim of this study was to evaluate the IOP values in a large group of children without systemic or ocular diseases and to investigate the effect of central corneal thickness (CCT) and radius of curvature on the IOP values measured. The cohort was an ethnically homogenous Polish population and three methods were used to collect IOP readings. The values for each method were compared with each other and with biometric data: $\mathrm{CCT}$ and radius of curvature.

\section{Methods}

The subject base consisted of 75 children (30 female and 45 male) with a low refractive error range $( \pm 2 \mathrm{D}$ (sphere); $\pm 0.5 \mathrm{D}$ (cylinder)), aged between 5 and 17 years (mean \pm standard deviation [SD] $11.3 \pm 3.0$ years) who presented for a regular eye examination. Measurements were made on 115 healthy eyes. Some of the subjects presented with ocular trauma in one eye and in such cases only the other, healthy, eye was included in the study. Only subjects who could comply with instructions given and from whom reliable measurements could be taken were used in this study. Subjects and their parents or guardians were fully informed of all procedures and why these were being conducted. In addition, subjects were carefully instructed, prior to each measurement, in what would be required of them during that procedure, the correct gaze position to adopt and, in the case of tonometry asked not to blink during the measurement. For measurements where contact with the eye was necessary, this was first demonstrated on the hand of the subject to indicate the level of contact that would be made on the eye.

Visual acuity was measured at distance and near with and without correction (BCVA). Radius of curvature was measured using an autorefraktometr Speedy-K (Righton). Three tonometers were used to obtain IOP: a non-contact tonometer (NCT) (Reichert, ophthalmic instrument XPERT NCT Plus Advanced Logic Tonometer), the ICare tonometer (TA01i, Icare Finland Oy) and the Goldmann applanation tonometer (GAT) fitted to a Nikon Slit Lamp NS-1 V. After measuring the radius of curvature, IOP was measured with the NCT and then with the ICare tonometer without anaesthetic drops. One drop of 0.5\% Alcaine (Proparacaine Hydrochloride, Alcon-Couvreur) was instilled into the examined eye prior to measuring CCT. One drop of $2 \%$ Thilorbin (Fluorescein Sodium, Oxybuprocaine Hydrochloride, Alcon Pharma $\mathrm{GmbH}$ ) was then put into the conjunctival sac, 30 seconds before the GAT measurement. Three NCT measurements, three ICare measurements and three GAT measurements were taken for each eye in the study. For each tonometer, values were averaged and the averages used for analysis. Variations in readings, for a given subject were NCT $( \pm 2 \mathrm{mmHg})$, ICare $( \pm 1 \mathrm{mmHg})$ and GAT $( \pm 1 \mathrm{mmHg})$. Measurements were rendered invalid if a subject blinked or moved their eye or head. At least quarter of an hour was taken between successive measurements with the different instruments in order to exclude any residual effect of previous measurements.

Six measurements of CCT were taken for each eye with an ultrasound pachymeter (Pacline Optikon 2000 OKB 181). The average values, within $\pm 5 \mu \mathrm{m}$, were accepted for analysis. All measurements were made between 9 am and 11 am.

Statistical analysis was conducted using STATISTICA 7 (StatSoft, Inc.). The three methods of IOP measurements were compared using the analysis of variance (ANOVA) and Tukey's HSD test. Equality of variances was tested using Brown-Forsythe and Levene's tests and the Pearson product-moment correlation coefficient $(r)$ was used to determine correlations between variables ( $r=0.7-0.99$ - high correlation, $r=0.4-0.69$ - medium correlation and $r<0.4$ - no correlation). Statistical significance was taken as $\mathrm{p}<0.05$.

The project was approved by the Ethics Committee of the Faculty of Ophthalmology at the Medical Academy in Wrocław and adhered to the Tenets of the Declaration of Helsinki. Informed parental consent and the assent of the subject were obtained before measurements were taken.

Table 1 Biometric and clinical data for the entire cohort, and for subgroups with central corneal thickness (CCT) < and $\geq 563 \mu \mathrm{m}$

\begin{tabular}{|c|c|c|c|}
\hline & $\frac{\text { All }}{\text { mean } \pm S D}$ & $\frac{\mathrm{CCT}<563 \mu \mathrm{m}}{\text { mean } \pm \mathrm{SD}}$ & $\frac{\mathrm{CCT} \geq 563 \mu \mathrm{m}}{\text { mean } \pm \mathrm{SD}}$ \\
\hline age [years] & $11.3 \pm 3.0$ & $11.9 \pm 2.8$ & $10.7 \pm 3.0$ \\
\hline CCT $[\mu \mathrm{m}]$ & $563 \pm 30$ & $540 \pm 18$ & $588 \pm 19$ \\
\hline $\mathrm{R}[\mathrm{mm}]$ & $7.85 \pm 0.29$ & $7.82 \pm 0.30$ & $7.87 \pm 0.28$ \\
\hline ICare $[\mathrm{mmHg}]$ & $16.9 \pm 3.4$ & $15.8 \pm 2.6$ & $18.1 \pm 3.7$ \\
\hline GAT [mmHg] & $14.7 \pm 2.9$ & $13.6 \pm 2.6$ & $15.8 \pm 2.8$ \\
\hline $\mathrm{NCT}[\mathrm{mmHg}]$ & $15.9 \pm 3.5$ & $14.4 \pm 3.0$ & $17.6 \pm 3.4$ \\
\hline
\end{tabular}



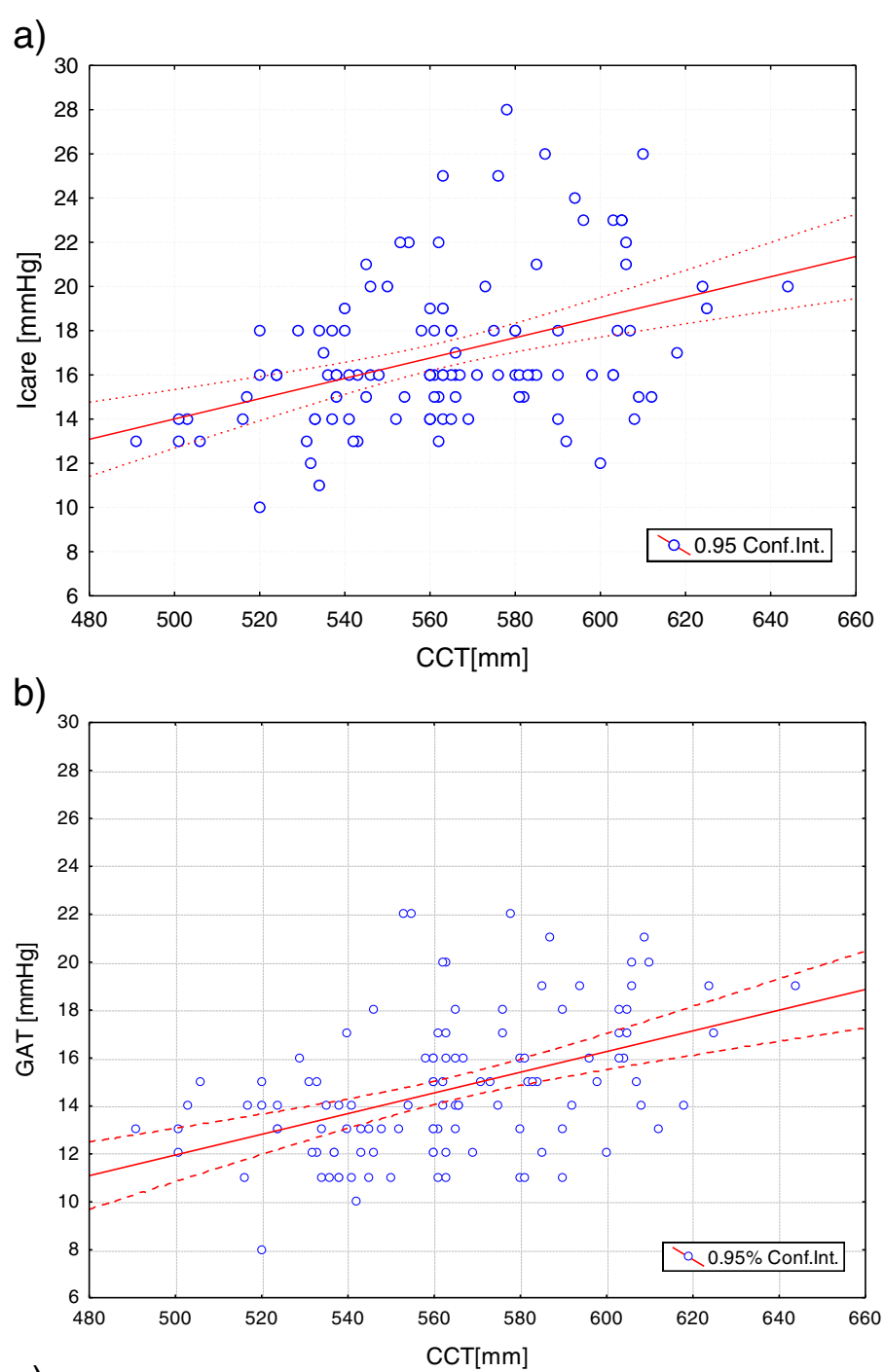

c)

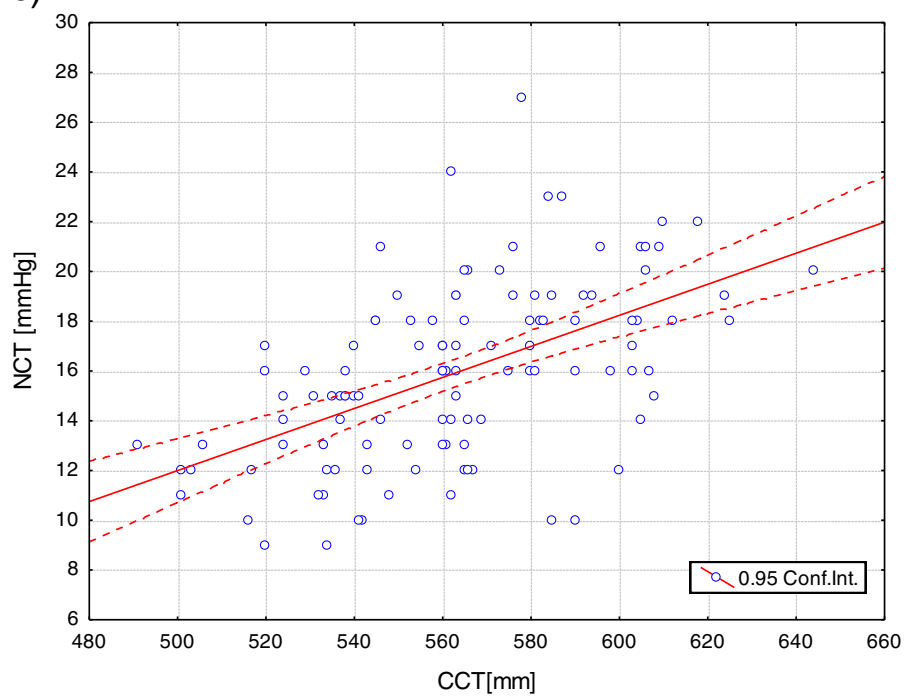

Figure 1 Intraocular pressure (IOP) plotted against central corneal thickness (CCT) $(\mu \mathrm{m})$ for IOP values obtained with the a) ICare tonometer; b) Goldmann applanation tonometer (GAT); c) non-contact tonometer (NCT). 


\section{Results}

Subject age, CCT, radius of curvature and IOP results, as measured with the three tonometers, are shown in Table 1 for the entire data set and for subsets with thinner and thicker corneae. No trend or correlation was found between age and IOP with any of the instruments.

The lowest IOP values were obtained with the GAT $(14.7 \pm 2.9 \mathrm{mmHg})$; the highest with the ICare tonometer $(16.9 \pm 3.4 \mathrm{mmHg})$. When the IOP results are plotted against the CCT (Figure 1), there is a positive linear trend that is steepest for the NCT. The variation of IOP with CCT was highly statistically significant for all three tonometers (ICare, $\mathrm{p}<0.001$; GAT, $\mathrm{p}<0.001$; NCT, $\mathrm{p}<0.001$ ). Whilst CCT is highly correlated with IOP, only IOP values obtained with ICare tonometer have a statistically significant correlation with radius of curvature $(\mathrm{p}<0.004)$.

To determine whether measurements of IOP obtained from the three instruments, differed significantly, comparative plots of the NCT and GAT, the ICare and the GAT and the NCT and ICare were plotted as shown in Figures 2a), b) and c) respectively. When confidence intervals (95\%) are compared for the whole cohort: NCT (CI 15.26-16.60), ICare (CI 16.26-17.54), GAT (CI 14.1315.23), a statistically significance difference, based on ANOVA and Tukey HSD testing was found between GAT and ICare $(\mathrm{p}<0.001)$ and GAT and NCT $(\mathrm{p}=0.01)$, but not between NCT and the ICare tonometer (Table 2).

In order to investigate the effect of CCT on the comparability of IOP measurements between different tonometers, the cohort was divided into two groups depending on CCT range: either side of the mean value of $563 \mu \mathrm{m}:<563 \mu \mathrm{m}$ (59 subjects) and $\geq 563 \mu \mathrm{m}$ (56 subjects) (Table 1). A slightly greater range of IOP values with the three tonometers is seen for the group with the thicker corneae. Comparison of confidence intervals (CI: GAT: 12.97-14.33; NCT: 13.58-15.14; ICare: 15.12-16.48) shows that for thinner corneae $(<563 \mu \mathrm{m})$, there is a statistically significant difference between IOP values taken with the GAT and ICare tonometer $(\mathrm{p}<0.001)$ and between those obtained with the NCT and ICare $(\mathrm{p}=0.02)$ but not between measurements obtained with GAT and NCT $(p=0.48)$ (Table 2). For the group with the higher $\mathrm{CCT}$, statistical significance is found between ICare and GAT $(\mathrm{p}<0.001)$ and GAT and NCT $(\mathrm{p}=0.01)$ but not between ICare and NCT $(\mathrm{p}=0.85)$ (Table 2) (CI: GAT: 14.12-15.22; NCT: 15.27-16.58; ICare: 16.38-17.54). There is no statistically significant correlation between radius of curvature and IOP values obtained with any instrument for the group with thinner corneae but a correlation between radius of curvature and IOP obtained with the ICare tonometer is found for the group with the thicker corneal values $(\mathrm{p}<0.008)$. For the cohort with thinner corneae, CCT was statistically significantly correlated with IOP obtained with the ICare tonometer and the NCT $(\mathrm{p}<0.006, \mathrm{p}<0.002$ respectively). Conversely, for the group with thicker corneae, correlation with CCT was found only with the GAT ( $<0.025)$.

The IOP measures obtained with the GAT can be corrected for thickness differences, using the Ehlers correction factor, an algorithm derived for the adult eye [14]. Applying the correction factor, does not alter the correlations between IOP values obtained with the different tonometers. For the entire cohort as well as for the subgroup with CCT $\geq 563 \mu \mathrm{m}$, statistically significant differences were found between IOP measurements obtained with the GAT and measurements obtained with both of the other instruments; for the subgroup with $\mathrm{CCT}<563 \mu \mathrm{m}$, measurements taken with ICare tonometer are statistically significantly different to those with either of the other instruments.

Figure 3 shows the confidence intervals for IOP values measured with the ICare tonometer, GAT (corrected and uncorrected) and for the NCT, for the entire cohort (Figure 3a), for the subgroup with $\mathrm{CCT}<563 \mu \mathrm{m}$ (Figure $3 \mathrm{~b}$ ) and for the subgroup with CCT $\geq 563 \mu \mathrm{m}$ (Figure 3c). It is notable that for the latter, there is a significant difference between the corrected and the uncorrected GAT values.

When the data are divided into lower and higher ranges of radius of curvature, taking the mean of $7.85 \mathrm{~mm}$ as the midpoint (Table 3), statistically significant differences are found only between IOP measurements obtained with the ICare instrument and the GAT for cohorts of both lower $(<7.85 \mathrm{~mm})$ and higher $(\geq 7.85 \mathrm{~mm}$ ) radii of curvature (Table 4).

The differences in IOP values obtained from the three tonometers were not normally distributed and hence Bland-Altman plots were not constructed. The proportion of measurements (as a percentage of the whole set) showing differences in magnitudes of IOP are shown plotted in Figure 4. For all three data pairs, in over $60 \%$ of measurements, differences in IOP values were $\leq 2 \mathrm{mmHg}$. Around $20 \%$ of the measurements show differences in IOP, between ICare and NCT, of $\geq 4 \mathrm{mmHg}$. This compares to $23 \%$ of measurements showing differences of such magnitude between GAT and NCT and $26 \%$ showing this magnitude of difference between the ICare tonometer and GAT. There was no trend or consistency in the magnitude of the differences for any pair of tonometers.

\section{Discussion}

A number of comparative analyses of tonometers have been conducted on adult eyes, both normal and glaucomatous or hypertensive [9-12]. Fewer studies have conducted similar comparative analyses with the eyes of 

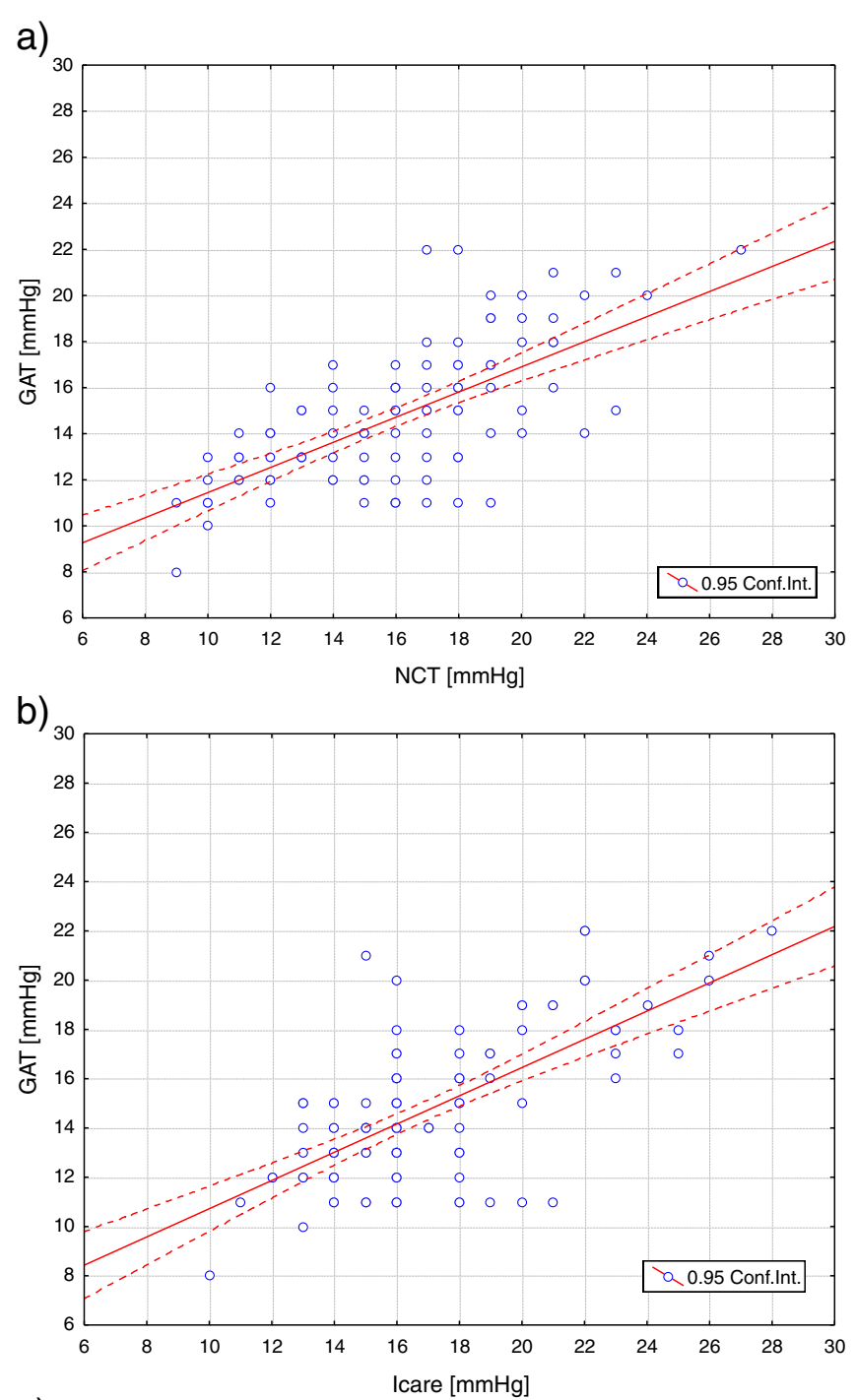

c)

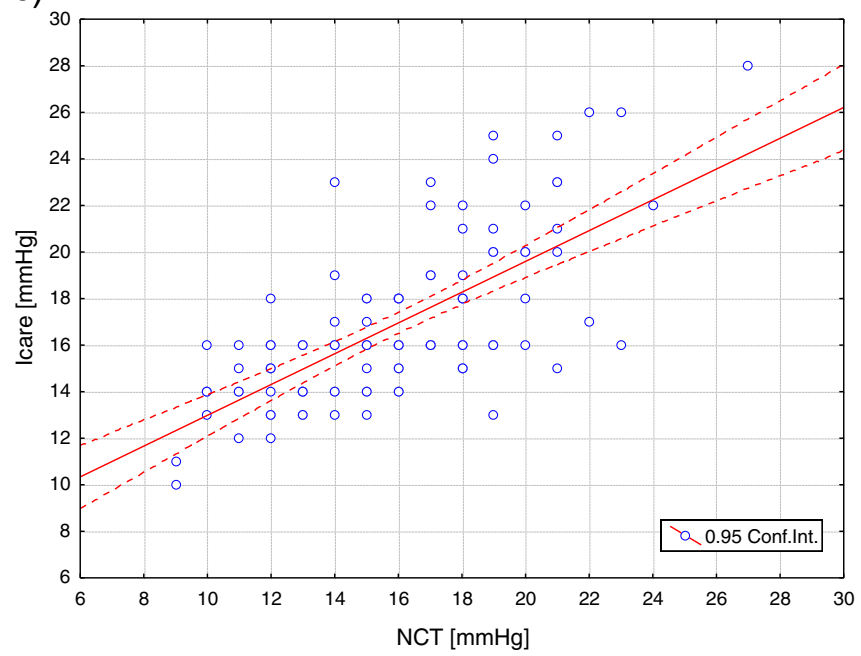

Figure 2 Intraocular pressure (IOP) obtained with a) Goldmann applanation tonometer (GAT) plotted against those obtained with the non-contact tonometer (NCT); b) Goldmann applanation tonometer (GAT) plotted against those obtained with the ICare tonometer; c) the ICare tonometer plotted against those obtained with the non-contact tonometer (NCT). 
children. The ICare tonometer was developed for use on patients who present with the sort of measurement problems that are often associated with children. The instrument is easy to handle, portable, light weight, and does not require anaesthesia or fluorescein. Some of these advantages are also pertinent to the NCT. Comparison of the ICare with an NCT on children from 6 months to 15 years of age showed comparability between instruments but also demonstrated the greater tolerance for measurements taken with the ICare tonometer compared with the NCT, on younger children [6]. High levels of tolerance for, as well as reproducibility of, IOP measurements were also found with the ICare tonometer, on a group of healthy infants [5] and schoolchildren [3]. Ease of use and tolerance, however, need to be balanced against effectiveness and accuracy. For many years, applanation tonometry was considered to set a standard against which other instruments were measured. However, recent reviews that have compared a number of instruments suggest that depending on the population and the application, different instruments may be preferred $[15,16]$.

A previous study of 460 children aged from 0 to 16 years, found that IOP increases from birth to around 7-8 years (slightly earlier for males than for females) and then stabilises [2]. The results of this study do not show any correlation or trends with age but the population used in this study was largely beyond the age range over which the rapid increase in IOP was observed [2].

The findings indicate that radius of curvature is correlated with IOP only for the ICare tonometer; this applies for the entire cohort and for the subgroup of subjects with thicker corneae. The only other comparable study, on Turkish schoolchildren, aged between 7 and 12 years, found no correlation between IOP and radius of curvature [4]. Significant differences between measurements obtained with the ICare tonometer and GAT that exist for the entire data set are evident for subsets of both lower and higher radii of curvature ranges. Given that only results obtained using the ICare tonometer showed a statistically significant correlation with radius of curvature and that the most significant differences between

Table 2 Correlations between tonometers: ICare, Goldmann applanation tonometer (GAT) and non-contact tonometer (NCT) for the entire cohort, and for subgroups with central corneal thickness $(C C T)<$ and $\geq 563 \mu \mathrm{m}$

\begin{tabular}{|c|c|c|c|}
\hline & All & $\mathrm{CCT}<563 \mu \mathrm{m}$ & $\mathrm{CCT} \geq 563 \mu \mathrm{m}$ \\
\hline & $p$ & $p$ & $p$ \\
\hline Icare vs. GAT & $<0.001$ & $<0.001$ & $<0.001$ \\
\hline Icare vs. NCT & 0.09 & 0.02 & 0.85 \\
\hline GAT vs. NCT & 0.01 & 0.48 & 0.01 \\
\hline
\end{tabular}

tonometers for the whole data set are evident between the ICare tonometer and GAT, it is not surprising that measurements vary significantly between these two instruments in cohorts with lower and higher radii of curvature ranges. Previous findings on adult eyes have found radius of curvature to influence readings with GAT [17] and with ICare (for CCT > $556 \mu \mathrm{m}$ ) [18].

Correlation with CCT and IOP was found for all tonometers used and CCT was found to affect correlations between the IOP values obtained with the different instruments. These correlations applied whether or not a correction factor for the GAT was used. This correction factor, derived for an adult eye [14], may not be appropriate for the eyes of children.

It should be noted that the extent of the effect of CCT on IOP measurements is not clear even for the adult eye. Originally, Ehlers and colleagues proposed a correction factor for CCT variations based on finding a significant correlation between CCT and IOP [14]. However, subsequent studies have not been consistent in their findings about how, and indeed whether at all, CCT should be corrected. Whilst a number of studies have found variations in IOP with CCT [19-23], correction factors have varied [14,23] and the use of any correction factor for the healthy adult cornea has been questioned $[24,25]$. Paucity in the understanding of the rheological properties of the cornea prevents definitive conclusions from being made about the effect of CCT on IOP measurements in the adult eye.

This notwithstanding, the findings in this study indicate that the thickness of the cornea in children can have an effect on correlation of IOP measurements between instruments. The ICare tonometer and the GAT gave readings that were significantly different statistically regardless of CCT; the NCT gave comparable readings with the ICare tonometer for the subgroup with thicker cornea and for the group as a whole and was comparable with the GAT for the subgroup of CCT $<563 \mu \mathrm{m}$.

This is the first study of IOP measurements on healthy Polish schoolchildren. It contributes to the literature on IOP in the young eye and provides a comparative study to those conducted on other ethnic groups. Findings from this study show that average CCT values were higher than those found in controls aged between 5-17 years from the USA (mean CCT $=555 \pm 37 \mu \mathrm{m}$ ) [26]. This included Caucasian and African ethnicities. For the Caucasian ('white') group alone, the average CCT was $564 \pm 28 \mu \mathrm{m}$, which is very close to the mean value found in this study (563 \pm $30 \mu \mathrm{m}$ ). The IOP for controls (Caucasian and African) was found to be $14.9 \pm 2.7 \mathrm{mmHg}$ for the Goldmann and 15.1 $\pm 2.4 \mathrm{mmHg}$ for the Tono-Pen [19]. This compares to 14.7 $\pm 2.9 \mathrm{mmHg}$ (GAT) in this paper. A study of 460 Italian subjects, aged 0 to 16 years of age, (with 282 subjects aged 


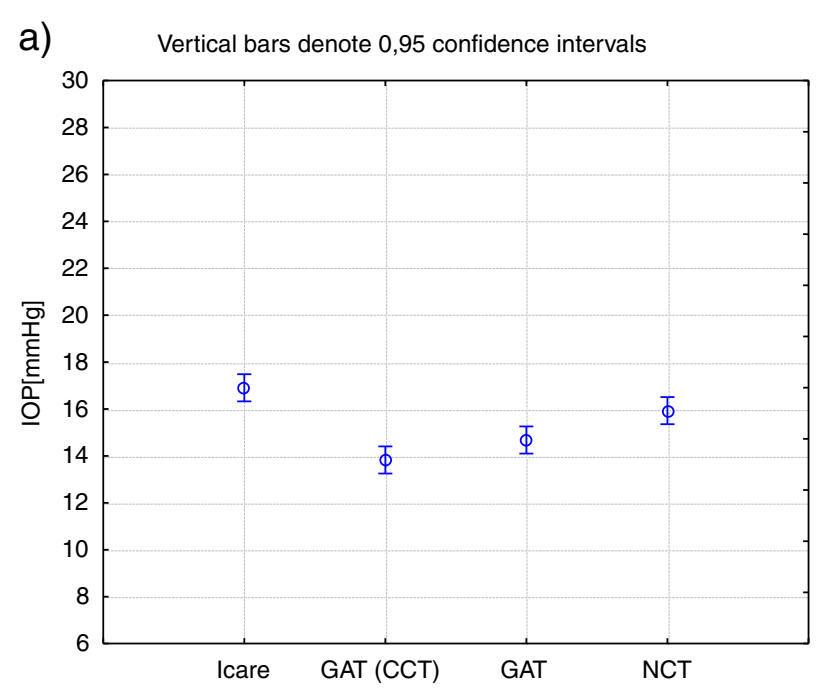

b) Vertical bars denote 0,95 confidence intervals

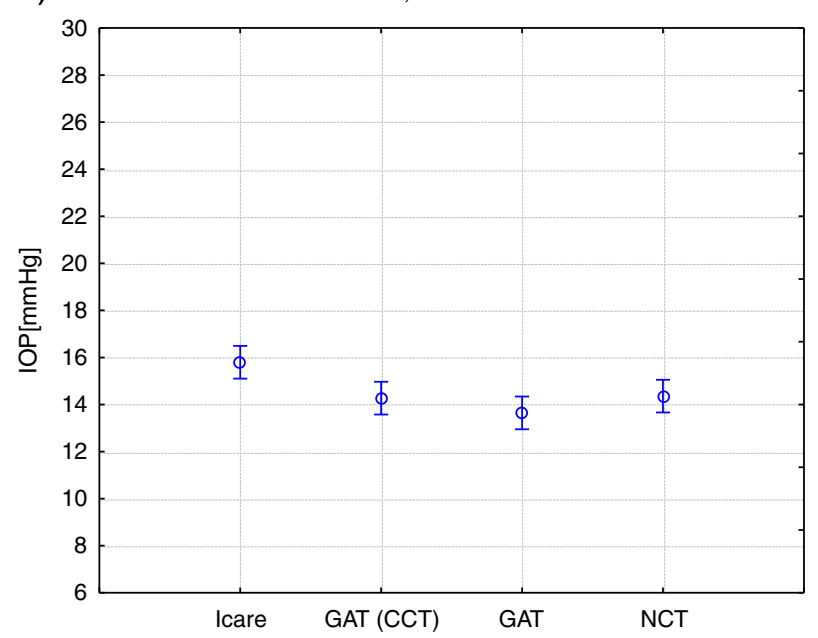

C) Vertical bars denote 0,95 confidence intervals

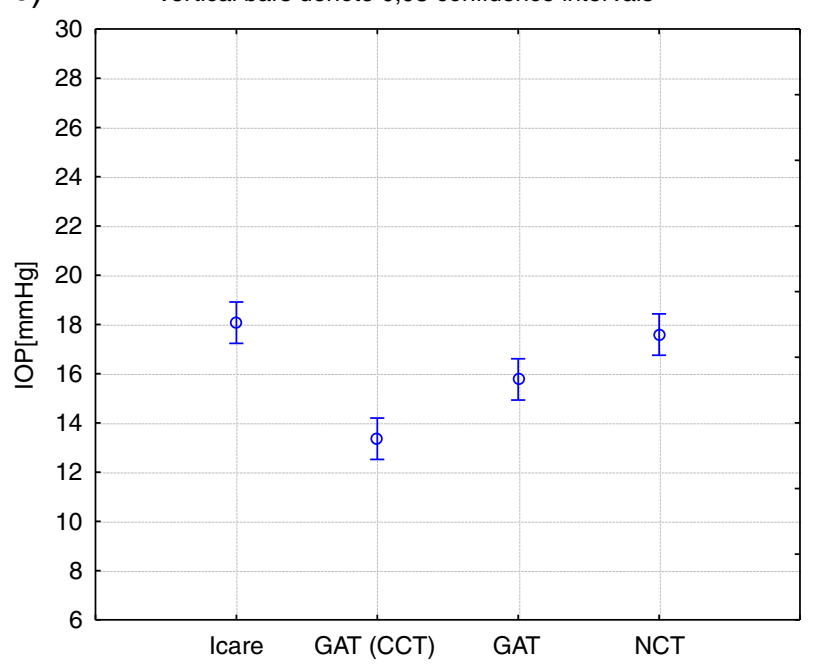

Figure 3 Confidence intervals for intraocular pressure values (IOP) $(\mathrm{mmHg})$ obtained with the ICare tonometer, Goldmann applanation tonometer (GAT) corrected and uncorrected and the non-contact tonometer (NCT) for a) the entire cohort; b) subgroup with CCT $<563 \mu \mathrm{m}$; c) subgroup with $\mathrm{CCT} \geq 563 \mu \mathrm{m}$. 
Table 3 Biometric and clinical data for the entire cohort, and for subgroups with corneal radius $(R)<$ and $\geq$ $7.85 \mathrm{~mm}$

\begin{tabular}{|c|c|c|c|}
\hline & $\frac{\text { All }}{\text { mean } \pm S D}$ & $\frac{\mathrm{R}<7.85 \mathrm{~mm}}{\text { mean } \pm \mathrm{SD}}$ & $\frac{\mathrm{R} \geq 7.85 \mathrm{~mm}}{\text { mean } \pm \mathrm{SD}}$ \\
\hline age [years] & $11.3 \pm 3.0$ & $11.4 \pm 3.0$ & $11.2 \pm 3.0$ \\
\hline $\mathrm{CCT}[\mu \mathrm{m}]$ & $563 \pm 30$ & $559 \pm 18$ & $568 \pm 17$ \\
\hline $\mathrm{R}[\mathrm{mm}]$ & $7.85 \pm 0.29$ & $7.63 \pm 0.15$ & $7.09 \pm 0.20$ \\
\hline ICare $[\mathrm{mmHg}]$ & $16.9 \pm 3.4$ & $15.9 \pm 2.8$ & $18.0 \pm 3.6$ \\
\hline GAT [mmHg] & $14.7 \pm 2.9$ & $14.3 \pm 2.4$ & $15.1 \pm 3.4$ \\
\hline NCT [mmHg] & $15.9 \pm 3.5$ & $15.5 \pm 3.3$ & $16.5 \pm 3.7$ \\
\hline
\end{tabular}

5-16 years), showed a mean IOP of $13.9 \pm 2.3 \mathrm{mmHg}$ and $14.88 \pm 2.39 \mathrm{mmHg}$ for $10-16$ year old males and females respectively using an NCT. These values are slightly lower than the IOP values found with the NCT in this study (15.9 $\pm 3.5 \mathrm{~mm} \mathrm{Hg}$, males and females combined) but are within the same range. No statistically significant differences between males and females were found in this study.

Previous investigations on 165 Turkish schoolchildren, aged 7-12 years, showed mean IOP values of $16.81 \pm$ $3.1 \mathrm{mmHg}$ using rebound tonometry which is very close to the $16.9 \pm 3.4 \mathrm{mmHg}$ found for the ICare in this study. IOP measurements on Japanese subjects aged from 6 months to 15 years of age reported mean IOP values of $15.9 \pm 2.3 \mathrm{mmHg}$ and $15.1 \pm 2.6 \mathrm{mmHg}$ in right and left eyes of 130 subjects respectively for the NCT with $15.1 \pm 2.6 \mathrm{~mm} \mathrm{Hg}$ and $13.9 \pm 2.9 \mathrm{mmHg}$ in right and left eyes respectively of 160 subjects for the ICare tonometer [6]. No explanation was given for the greater difference between right and left eyes using the latter instrument but the authors indicate that ICare is the more suitable instrument for children under 6 years of age [6].
Table 4 Correlations between tonometers: ICare, Goldmann applanation tonometer (GAT) and non-contact tonometer (NCT) for the entire cohort, and for subgroups with corneal radius $(R)<$ and $\geq 7.85 \mathrm{~mm}$

\begin{tabular}{|c|c|c|c|}
\hline & All & $\mathrm{R}<7.85 \mathrm{~mm}$ & $\mathrm{R} \geq 7.85 \mathrm{~mm}$ \\
\hline & p & $\mathrm{p}$ & p \\
\hline Icare vs. GAT & $<0.001$ & 0.009 & $<0.001$ \\
\hline Icare vs. NCT & 0.09 & 0.85 & 0.07 \\
\hline GAT vs. NCT & 0.01 & 0.09 & 0.17 \\
\hline
\end{tabular}

A study that uses young subjects, notwithstanding the steps taken to explain the procedure and to take carefully controlled measurements, can be limited by the extent of subject participation and parental involvement. Collaboration of subjects, such as children, is greatly improved if the subjects feel comfortable in the environment where measurements are taken and if there is no or minimal parental pressure and/or anxiety. The University clinic where the study was conducted is one which routinely deals with large numbers of young subjects. It is purposely equipped to create an appropriate ambience and a relaxed atmosphere for these age groups. In addition, the subjects who participated in this study were all familiar with the clinic, parents/guardians had confidence in the investigators rendering high both subject and parental levels of cooperation.

The differences in IOP measurements from the three tonometers, presented in pairs (Figure 4), varied, for most measurements taken, by up to $2 \mathrm{mmHg}$. However, from a fifth to a quarter of the measurements varied by $\geq 4 \mathrm{mmHg}$. As there is no gold standard for tonometry measurement in children it is not possible to say which measurement can be taken as the most reliable. BlandAltman plots, which are commonly used to check agreement between methods, often depend on one technique

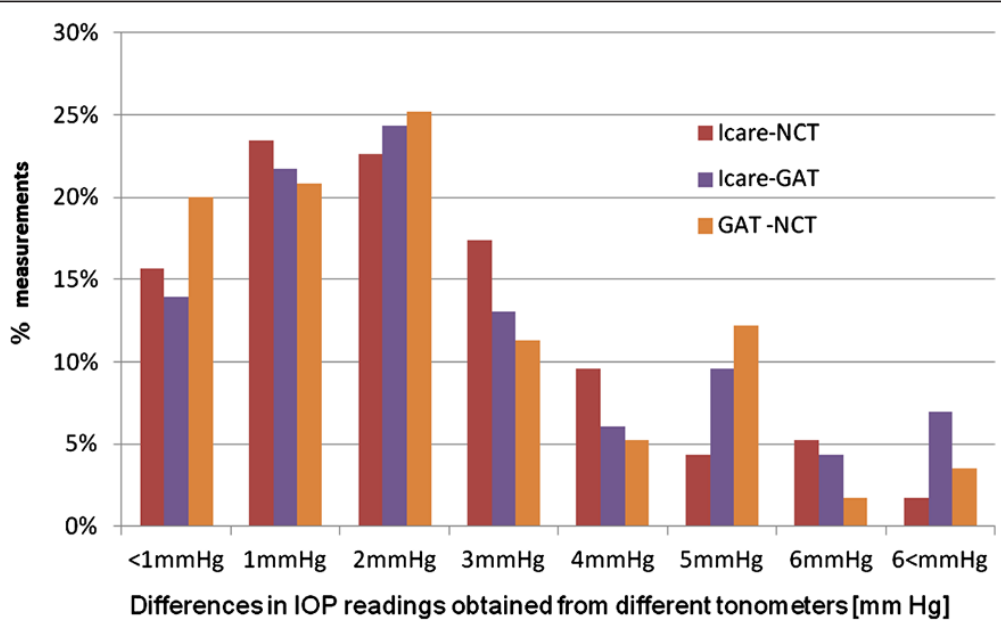

Figure 4 Percentage of measurements plotted against varying differences in IOP readings between the three instruments tested 
being the standard against which the other is measured [27]. Given the effect of CCT on IOP measurements and the paucity of knowledge about the growing eye or how to correct or adjust for CCT in such cases, it is prudent to avoid reliance on any one method. Many more studies on children from different cohorts are required before consistent trends can be established and expected agerelated norms derived.

\section{Conclusions}

There is still no universally accepted means of determining IOP in the growing eye. Three tonometers, used to obtain IOP values on a group of Polish children aged 5-17 years (mean \pm SD $11.3 \pm 3.0$ years), show statistically significant differences in measurements. The ICare tonometer provides statistically higher IOP values than the GAT whilst correlations with the NCT and the other two instruments are affected by corneal thickness. Further studies on cohorts of children from different ethnic groups and using a range of instruments are needed before it is possible to determine which instrument is best for a growing eye and before a normative scale of IOP values for different stages of growth can be derived.

\section{Competing interests}

The authors declare that they have no competing interests.

\section{Authors' contributions}

All authors conceived of and designed the experimental protocol. MAW and PKB collected the data. All authors were involved in the analysis. BP wrote the first draft of the manuscript. MAW and BP reviewed and revised the manuscript and produced the final version. All authors read and approved the final manuscript.

\section{Acknowledgements}

The authors acknowledge with gratitude funding received from the Wroclaw Medical University (Grant no 1933).

This project was funded by the Wroclaw Medical University (Grant no 1933).

\section{Author details}

'Department of Ophthalmology, Wroclaw Medical University, Borowska 213 50-556, Wroclaw, Poland. 'Institute of Physics, Wroclaw University of Technology, Wybrzeze Wyspianskiego 27, 50-370, Wroclaw, Poland. ${ }^{3}$ Faculty of Science, Engineering and Computing, Kingston University, (Roehampton Vale Campus), Friars Avenue, London SW15 3DW, UK.

Received: 25 April 2012 Accepted: 23 November 2012

Published: 2 December 2012

\section{References}

1. Duckman RH: A new induction-based impact method for measuring intraocular pressure. Acta Ophthalmologica Scandinavica 2000, 78:142-145.

2. Pensiero S, Da Pozzo S, Perissutti P, Cavallini GM, Guerra R: Normal intraocular pressure in children. J Pediatric Ophthalmol \& Strabismus 1992, 29:79-84.

3. Sahin A, Basmak H, Niyaz L, Yildirim N: Reproducibility and tolerability of the ICare rebound tonometer in school children. J Glaucoma 2007, 16:185-188.

4. Sahin A, Basmak H, Yildirim N: The influence of central corneal thickness and corneal curvature on intraocular pressure measured by Tono-Pen and rebound tonometer in children. J Glaucoma 2008, 17:57-61.

5. Lundvall A, Svedberg H, Chen E: Application of the ICare rebound tonometer in healthy infants. J Glaucoma 2011, 20:7-9.
6. Kageyama M, Hirooka K, Baba T, Shiraga F: Comparison of ICare rebound tonometer with noncontact tonometer in healthy children. J Glaucoma 2011, 20:63-66.

7. Brusini P, Salvetat ML, Zeppieri M, Tosoni C, Parisi L: Comparison of ICare tonometer with goldmann applanation tonometer in glaucoma patients. J Glaucoma 2006, 15:213-217.

8. Davies LN, Bartlett H, Mallen EA, Wolffsohn JS: Clinical evaluation of rebound tonometer. Acta Ophthalmol Scand 2006, 84:206-209.

9. Martinez-de-la-Casa JM, Garcia-Feijoo J, Vico E, Fernandez-Vidal A, del Benitez Castillo JM, Wasfi M, Garcia-Sanchez J: Effect of corneal thickness on dynamic contour, rebound, and Goldmann tonometry. Ophthalmology 2006, 113:2156-2162.

10. Nakamura M, Darhad U, Tatsumi Y, Fujioka M, Kusuhara A, Maeda H, Negi A: Agreement of rebound tonometer in measuring intraocular pressure with three types of applanation tonometers. Am J Ophthamol 2006, 142:332-334.

11. Pakrou N, Gray T, Mills RA, Landers JA, Craig J: Clinical comparison of the ICare tonometer and Goldmann applanation tonometry. J Glaucoma 2008, 17:43-47.

12. Rehnman JB, Martin L: Comparison of rebound and applanation tonometry in the management of patients treated for glaucoma or ocular hypertension. Ophthal Physiol Opt 2008, 28:382-386.

13. Van Der Jagt $L H$, Jansonius NM: Three portable tonometers, the TGDc-01, the ICare and the Tonopen XL, compared with each other and with Goldmann applanation tonometry. Ophthal Physiol Opt 2005, 25:429-435.

14. Ehlers N, Bramsen T, Sperling S: Applanation tonometry and central corneal thickness. Acta Ophthalmol 1975, 53:34-43.

15. Kniestedt C, Punjabi O, Lin S, Stamper RL: Tonometry through the ages. Surv Ophthalmol 2008, 53:568-591.

16. Chihara E: Assessment of true intraocular pressure: the gap between theory and practical data. Surv Ophthalmol 2008, 53:203-218.

17. Krzyzanowska-Berkowska P, Asejczyk-Widlicka M: Clinical evaluation of the ICare tonometer in measuring intraocular pressure. Klin Ocz 2010, $112: 217-220$.

18. Harada Y, Hirose N, Kubota T, Tawara A: The influence of central corneal thickness and corneal curvature radius on the intraocular pressure as measured by different tonometers: noncontact and goldmann applanation tonometers. J. Glaucoma 2008, 17:619-625.

19. Johnson M, Kass MA, Moses RA, Grodzki WJ: Increased cornea thickness simulating elevated intraocular pressure. Arch Ophthalmo/ 1978, 96:664-665.

20. Whitacre MM, Stein RA, Hassanein K: The effect of corneal thickness on applanation tonometry. Am J Ophthalmol 1993, 115:592-596.

21. Bhan A, Browning AC, Shah S, Hamilton R, Dave D, Dua HS: Effect of thickness on intraocular pressure measurements with the pneumotonometer, Goldmann applanation tonometer, and tono-pen. Invest Ophthalmol and Vis Sci 2002, 43:1389-1392.

22. Broman AT, Congdon NG, Bandeen-Roche K, Quigley HA: Influence of corneal structure, corneal responsiveness, and other ocular parameters on tonometric measurement of intraocular pressure. J Glaucoma 2007, 16:581-588

23. Stodtmeister R: Applanation tonometry and correction according to corneal thickness. Acta Ophthalmol Scand 1998, 76:319-324.

24. Feltgen N, Leifert D, Funk J: Correlation between central corneal thickness, applanation tonometry, and direct intracameral IOP readings. Br J Ophthalmol 2001, 85:85-87.

25. Pache M, Wilmsmeyer S, Lautebach S, Funk J: Dynamic contour tonometry versus Goldmann applanation tonometry: a comparative study. Graefe's Arch Clin Exp Ophthalmol 2005, 243:763-767.

26. Muir KW, Jin J, Freedman SF: Central corneal thickness and its relationship to Intraocular pressure in children. Ophthalmology 2004, 111:2220-2223.

27. Bland JM, Altman DG: Statistical methods for assessing agreement between two methods of clinical measurement. Lancet 1986, i:307-310.

doi:10.1186/1471-2415-12-61

Cite this article as: Krzyżanowska-Berkowska et al:: Intraocular pressure in a cohort of healthy eastern European schoolchildren: variations in method and corneal thickness. BMC Ophthalmology 2012 12:61. 